\title{
SYNTHESIS OF OPTICALLY ACTIVE 2-[4-(6-CHLORO-2-BENZOXAZOLYLOXY) PHENOXY]PROPIONAMIDE DERIVATIVES OF POTENTIAL HERBICIDE ACTIVITY
}

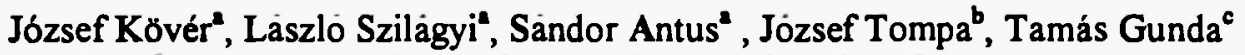 \\ Department of Organic Chemistry, University of Debrecen, \\ H-4010 Debrecen, P.O.Box 20 \\ ${ }^{b}$ ICN Hungary Ltd., H-4440 Tiszavasvari P.O.Box 1 \\ ' Research Group of Antibiotics of the Hungarian Academy of Sciences, \\ University of Debrecen, H-4010 Debrecen, P.O.Box 70
}

\begin{abstract}
An efficient two steps procedure has been developed for the synthesis of (R)-(+)-2-[4-(6chloro-2-benzoxazolyloxy)phenoxy]propionamides (8a-I) starting from (R)-(+)-methyl 2-(4-hydroxyphenoxy)-propionate (4). Treatment of $R-(+)-2$ with the primary amines $5 \mathbf{a}-\mathbf{f}$ resulted in $9 \mathrm{a}-\mathbf{f}$ quantitatively instead of a desired amidation $[R-(+)-2 \rightarrow R-(+)-8 a-f]$. This unexpected transformation was discussed on the basis of $\mathrm{QM}$ calculation.
\end{abstract}

\section{Introduction}

The 2-[(4-aryloxy or heteroaryloxy)phenoxy]propionic acid derivatives are widely used as readily available starting materials for the synthesis of biologically active substances (1-3) and plant growth regulators (4-10). Several of them, such as methyl 2-[4-(2,4-dichlorophenoxy)phenoxy] propionate (1), and ethyl 2-[4-(6-chloro-2-benzoxazolyloxy)phenoxy]propionate (2), with the trade names Diclofop-methyl ${ }^{R}$, and Fenoxaprop-ethyl ${ }^{R}$, respectively, have been introduced to the herbicidal market.<smiles>[R]OC(=O)[C@H](C)Oc1ccc(O[R])cc1</smiles>

\begin{tabular}{c|cl} 
& $\mathrm{R}^{\mathbf{l}}$ & $\mathrm{R}^{2}$ \\
\hline $\mathbf{1}$ & 2,4-dichlorophenyl & $\mathrm{Me}$ \\
2 & 6-chloro-2-benzoxazolyl & $\mathrm{Et}$ \\
3 & $\mathrm{H}$ & $\mathrm{Et}$
\end{tabular}

For the synthesis of these compounds two routes have been described in the literature; (a) the corresponding 4-aryloxy- or heteroaryloxyphenol derivative is reacted with a propionic acid derivative carrying a good leaving group (such as a halogene, tosyl or mesyl group) at C-2 under basic conditions to give the target phenoxypropionic acid derivative, or (b) alkylation of a 2-(4hydroxyphenoxy)-propionic acid derivative (prepared from hydroquinone with the above mentioned 
2-substituted alkylpropionates) with the corresponding halogene-substituted aromatic or heteroaromatic compounds also leads to the target molecule.

Although these strategies have some disadvantages but both are still widely used for the production of these types of herbicides on industrial scale. For example, Fenoxaprop-ethyl ${ }^{\mathbf{R}}$ (rac-2) is produced on route (b) from ethyl 2-(4-hydroxyphenyl)-propionate (rac-3) and 2,6-dichlorobenzoxazole (7). It should be also noted that its dextrorotatory enantiomer [(R)-(+)-2] is twice as active in biological tests as the racemate (11).

In continuation of our research program aimed at the development of new bioactive 2substituted benzoxazoles (12), we set our sights on the synthesis of (R)-(+)-2[4-(6-chloro-2benzoxazolyloxy)phenoxy]propionamides $(8 \mathrm{a}-\mathrm{f})$ to get further information about the structurebiological activity relationship concerning the herbicidal activity of Fenoxaprop derivatives.

\section{Results and discussion}

It seemed interesting to react the easily available optically pure (R)-(+)-methyl 2-(4hydroxyphenoxy)-propionate 4 with the primary amines 5 a-f at their boiling temperature without solvent in order to produce the amides (R)-(+)-6a-f of high optical purity and in good yield. Indeed, the amides (R)-(+)-6a-f could be obtained without loss of the optical purity of $(R)-(+)-3$ (Scheme 1 , Table 1). In the next step the amides (R)-(+)-6a-f were treated with 2,6-dichlorobenzoxazole (7) [prepared according to the literature (13)] in the presence of potassium carbonate in acetonitrile at $81^{\circ} \mathrm{C}$ to result in the amides (R)-(+)-8a-f of high optical purity $(>98 \%)$ in good yield (Table 2 ).

Scheme 1 .<smiles>[R]NC(=O)[C@H](C)Oc1ccc(O)cc1</smiles><smiles>[R]NC(=O)C(C)Oc1ccc(Oc2nc3ccc(Cl)cc3o2)cc1</smiles>

\begin{tabular}{r|l}
$5,6,8$ & \multicolumn{1}{|c}{$R$-(+)-8a-f } \\
\hline a & cyclopropyl \\
b & cyclopropylmethyl \\
c & 3,4-methylenedioxybenzyl \\
d & 2-pyridylmethyl \\
e & 3-pyridylmethyl \\
f & 4-pyridylmethyl
\end{tabular}


Although cleavage of 6-chloro-2-phenoxybenzoxazol to 6-chloro-2-(alkylamino)benzoxazoles by aliphatic primary amines has been reported in our previous paper (12), it seemed to be obvious to attempt the direct transformation of (R)-( +$)-2$ into the amides $(R)-(+)-8 a-f$ by using the corresponding primary amines (5a-f) both as solvent and reagent under mild conditions.

Therefore, (R)-(t)-2 was treated with 6 equivalents of the aliphatic primary amines 5a-f at room temperature and the reaction was followed by TLC. A very fast transformation of (R)-(+)-2 could be recognised (1-15 min.) in all cases but the cleavage of the 6-chloro-2-phenoxybenzoxazol moiety resulting quantitatively in 9a-f (12) occurred (Scheme 2) instead of the expected amidation $[(R)-(+)-2 \rightarrow(R)-(+)-8 a-1]$.

Scheme 2.<smiles>[R16]Nc1nc2ccc(Cl)cc2o1</smiles>

In order to shed more light on the unexpected reaction of $(R)-(+)-2$ with $5 a-f$, we performed simple QM calculations trying to find theoretical backgrounds to the high selectivity of C-2" and C1 toward nucleophylic reagents.

\begin{tabular}{|c|c|c|}
\hline Atom & Net charge & $\begin{array}{c}\text { Nucleophilic } \\
\text { superdelocalizability }\end{array}$ \\
\hline 1" & -0.0873 & 0.54882 \\
\hline 2" & 0.1888 & 1.37260 \\
\hline 3" & -0.174 & 0.74211 \\
\hline 4". & -0.0338 & 1.14009 \\
\hline $5 "$ & -0.1110 & 1.0591 \\
\hline 6" & -0.1214 & 1.30484 \\
\hline 7" & -0.0772 & 1.16639 \\
\hline $1^{\prime}$ & 0.0888 & 1.20725 \\
\hline $2^{\prime}$ & -0.1574 & 1.07269 \\
\hline $3^{\prime}$ & -0.0616 & 1.13223 \\
\hline $4^{\prime}$ & 0.0128 & 1.19688 \\
\hline $5^{\prime}$ & -0.0618 & 1.12261 \\
\hline $6^{\prime}$ & -0.1163 & 1.05231 \\
\hline 1 & 0.3641 & 1.09021 \\
\hline 2 & 0.0781 & 0.92311 \\
\hline 3 & -0.1570 & 0.68835 \\
\hline
\end{tabular}

Table 3. Calculated net charges and superdelocalizabilities of $R-(+)-2$.
The MOPAC package (PM3 Hamiltonian) implanted into the CAChe program (14) was used to calculate atomic properties. Simple net atomic charges cannot adequately describe the differences in experimental reactivities of C-2", C-1 and other atoms as depicted in Table 3. Superdelocalizability were used more successfully, since it puts more emphasis on the frontier orbital densities and the HOMOLUMO energies and reflects better the relative differences in reactivity of the different parts of the molecule. The data in Table 3 clearly show that C-2" is more susceptible to nucleophilic attack then the C-1 carbonyl group. 
In summary, our present results offer a simple and efficient method for the synthesis of the (R) $(+)-$ [4(6-chloro-2-benzoxazolyloxy)phenoxy]propionamides ( $\mathrm{R})-(+)-8 a-f$ whose herbicidal activity is currently under investigation.

\section{Experimental}

Melting points were determined on a Büchi 535 apparatus and are not corrected. ${ }^{1} \mathrm{H}-\mathrm{NMR}$ spectra were obtained on a Varian Gemini $200 \mathrm{NMR}$ spectrometer in $\mathrm{CDCl}_{3}$ and $\mathrm{DMSO}-\mathrm{d}_{6}$ (marked by an asterisk*) with TMS as internal standard. EI-MS spectra were obtained with a VG TRIO-2 instrument. TLC was performed on Merck Kieselgel $60 \mathrm{~F}_{254}$ pre-coated aluminum plates. The purity of the products was determined on a HP 1090 liquid-chromatograph and it has been found to be over 99.5\%. Column: Hypersil MOS (100x4.6 mm), solvent: (A) $0.01 \mathrm{~N}$ potassium dihydrogensulphate with $0.1 \%(\mathrm{v} / \mathrm{v}) \mathrm{H}_{3} \mathrm{PO}_{4}(99.99 \%)$ and $10 \%(\mathrm{v} / \mathrm{v})$ methanol; (B) methanol. The gradient was increased from $10 \%$ B to $75 \%$ B with a flow rate of $0.5 \mathrm{ml} / \mathrm{min}$. Optical rotation was measured with a Perkin-Elmer 241 polarimeter at the sodium-D line. The optical purity of the compounds was determined by NMR (Bruker WP 200 SY) using tris[3-(trifluoromethylhydroxymethylene)-dcamphorato]europium(III) as chiral shift reagent and it has been found to be over $98 \%$. Elemental analyses of 6a-f and 8a-f were carried out with a Carlo Erba 1106 analyser and the results were found to be within $\pm 0.4 \%$.

General procedure for the preparation of the (R)-(+)-[4-hydroxyphenoxy]propionamides [(R)(+)-6a-f].

A mixture of (R)-(+)-4 (15) $(9.80 \mathrm{~g}, 0.05 \mathrm{~mol}, \mathrm{ee} \%=98.9)$ and the primary amines $5 \mathrm{a}-\mathrm{f}(0.3$ mol) was heated with stirring at the boiling temperature of the amine under nitrogen atmosphere until no starting material could be detected by TLC. Then the excess of the amines was evaporated in vacuo. The residue was dissolved in chloroform $(50 \mathrm{~mL})$ and subsequently washed with $0.5 \mathrm{~N}$ hydrochloric acid $(2 \times 15 \mathrm{~mL})$, saturated sodium hydrocarbonate $(3 \times 10 \mathrm{~mL})$ and water $(3 \times 20$ $\mathrm{mL})$. The organic layer was dried over $\mathrm{MgSO}_{4}$, and evaporation gave the crude product whose purification on a silicagel column $(6 a, c, f: n$-hexane/acetone $=1: 1,6 b: n$-hexane/acetone $=2: 1,6 c$ : benzene:methanol $=4: 1$ ) yielded solid products (Table 1 ).

General procedure for the preparation of the (R)-(+)-2[4-(6-chloro-2-benzoxazolyloxy)phenoxy]propion amides $[R-(+)-8 \mathrm{a}-1]$.

A mixture of (R)-(+)-6a-f (30 mmol), 2,6-dichlorobenzoxazole (7) $(6.20 \mathrm{~g}, 33 \mathrm{mmol})$ and 
Table 1. Characterization of Compound (R)-(+)-6a-f

\begin{tabular}{|c|c|c|c|c|c|}
\hline Comp. & $\begin{array}{l}\text { React. } \\
\text { time (h) }\end{array}$ & $\begin{array}{l}\text { Yield } \\
(\%)\end{array}$ & $\begin{array}{l}\text { M. p. } \\
\text { (C) }\end{array}$ & $\begin{array}{l}{[\alpha]^{2 S}{ }_{D} C=1 \%} \\
\text { (solvent) }\end{array}$ & $\begin{array}{c}\text { Characteristic } \mathrm{H} \text { NMR data } \\
(\delta=\mathrm{ppm}, \mathrm{J}=\mathrm{Hz})\end{array}$ \\
\hline $6 a$ & 8 & 84 & 98 & $\begin{array}{c}15.8 \\
\left(\mathrm{CHCl}_{3}\right)\end{array}$ & $\begin{array}{l}1.50(\mathrm{~d}, \mathrm{~J}=7.35,3 \mathrm{H}, \mathrm{Me}), 4.52(\mathrm{q}, \mathrm{J}=7.35 \\
\mathrm{lH}, 2-\mathrm{H}), 6.65(\mathrm{bs}, \mathrm{lH}, \mathrm{NH})\end{array}$ \\
\hline $6 b$ & 10 & 78 & $82-83$ & $\begin{array}{c}15.4 \\
(\mathrm{MeOH})\end{array}$ & $\begin{array}{l}1.55(\mathrm{~d}, \mathrm{~J}=8.10,3 \mathrm{H}, \mathrm{Me}), 4.55(\mathrm{q}, \mathrm{J}=8.10, \\
\mathrm{lH}, 2-\mathrm{H}), 6.56(\mathrm{bs}, \mathrm{lH}, \mathrm{NH})\end{array}$ \\
\hline $6 c$ & 4.5 & 87 & 78-79 & $\begin{array}{c}23.0 \\
\left(\mathrm{CHCl}_{3}\right)\end{array}$ & $\begin{array}{l}1.56(\mathrm{~d}, \mathrm{~J}=7.25,3 \mathrm{H}, \mathrm{Me}), 4.35(\mathrm{q}, \mathrm{J}=6.68, \\
\left.2 \mathrm{H}, \mathrm{NHCH} \mathrm{H}_{2} \mathrm{Ar}\right), 4.60(\mathrm{q}, \mathrm{J}=7.25, \mathrm{lH}, 2-\mathrm{H}) \\
6.81(\mathrm{bs}, \mathrm{lH}, \mathrm{NH})\end{array}$ \\
\hline $6 d$ & 7.5 & 85 & $96-97$ & $\begin{array}{c}23.1 \\
\left(\mathrm{CHCl}_{3}\right)\end{array}$ & $\begin{array}{l}1.54(\mathrm{~d}, \mathrm{~J}=6.78,3 \mathrm{H}, \mathrm{Me}), 4.50-4.65(\mathrm{~m}, 3 \mathrm{H}, \\
\left.2-\mathrm{H} \text { and pyridyl- } \mathrm{CH}_{2}\right), 6.80(\mathrm{bs}, \mathrm{lH}, \mathrm{NH})\end{array}$ \\
\hline $6 e$ & 5.5 & 72 & $141-142$ & $\begin{array}{c}15.9 \\
\left(\mathrm{CHCl}_{3}\right)\end{array}$ & $\begin{array}{l}1.45(\mathrm{~d}, \mathrm{~J}=6.20,3 \mathrm{H}, \mathrm{Me}), 4.30(\mathrm{~d}, \mathrm{~J}=6.20 \\
\left.2 \mathrm{H}, \text { pyridyl } \mathrm{CH}_{2}\right), 4.42(\mathrm{q}, \mathrm{J}=6.20 \mathrm{lH}, 2-\mathrm{H}) \\
8.65(\mathrm{bs}, \mathrm{lH}, \mathrm{NH})\end{array}$ \\
\hline $6 f$ & 3.5 & 81 & $191-192$ & $\begin{array}{c}14.2 \\
(\mathrm{MeOH})\end{array}$ & $\begin{array}{l}1.45(\mathrm{~d}, \mathrm{~J}=6.80,3 \mathrm{H}, \mathrm{Me}), 4.30(\mathrm{~d}, \mathrm{~J}=6.20 \text {, } \\
\left.2 \mathrm{H}, \text { pyridyl } \mathrm{CH}_{2}\right), 4.60(\mathrm{q}, \mathrm{J}=6.80 \mathrm{lH}, 2-\mathrm{H}) \text {, } \\
8.66(\mathrm{t}, \mathrm{J}=4-5,1 \mathrm{H}, \mathrm{NH})\end{array}$ \\
\hline
\end{tabular}

Table 2. Characterization of Compound (R)-(+)-8a-f

\begin{tabular}{|c|c|c|c|c|c|}
\hline Comp. & $\begin{array}{l}\text { React. } \\
\text { time (h) }\end{array}$ & $\begin{array}{l}\text { Yield } \\
(\%)\end{array}$ & $\begin{array}{l}\text { M. p. }\left({ }^{\circ} \mathrm{C}\right) \\
\text { (solvent) }\end{array}$ & $\begin{array}{c}{[\alpha]^{25} C=1 \%} \\
\text { (solvent) }\end{array}$ & $\begin{array}{c}\text { Characteristic }{ }^{\prime} \mathrm{H} N M R \text { and MS data } \\
\left(\delta=p_{p m}, \mathrm{~J}=\mathrm{H}, \mathrm{e} / \mathrm{z} \%\right)\end{array}$ \\
\hline $8 a$ & 2 & 68 & $\begin{array}{l}188-189 \\
\text { (EtOH) }\end{array}$ & $\begin{array}{c}13.4 \\
\left(\mathrm{CHCl}_{3}\right)\end{array}$ & $\begin{array}{l}1.60(\mathrm{~d}, \mathrm{~J}=6.86,3 \mathrm{H}, \mathrm{Me}), 4.65(\mathrm{q}, \mathrm{J}=7.35 \\
\mathrm{lH}, 2-\mathrm{H}), 6.60(\mathrm{bs}, \mathrm{lH}, \mathrm{NH}) \\
372\left(\mathrm{M}^{+}, 9\right), 288(20), 261(20), 112(95) \\
84(100)\end{array}$ \\
\hline $8 b$ & 1.5 & 88 & $\begin{array}{l}157-158 \\
\left(\mathrm{CH}_{3} \mathrm{CN}\right)\end{array}$ & $\begin{array}{c}13.6 \\
\left(\mathrm{CHCl}_{3}\right)\end{array}$ & $\begin{array}{l}1.61(\mathrm{~d}, \mathrm{~J}=7.55,3 \mathrm{H}, \mathrm{Me}), 4.56(\mathrm{q}, \mathrm{J}=7.55 \\
\mathrm{lH}, 2-\mathrm{H}), 6.50(\mathrm{bs}, \mathrm{lH}, \mathrm{NH}) \\
386\left(\mathrm{M}^{+}, 10\right), 288(10), 261(18), 126(52) \\
98(42), 55(100)\end{array}$ \\
\hline $8 c$ & 10 & 67 & $\begin{array}{c}158-159 \\
\left(\mathrm{C}_{6} \mathrm{H}_{6}\right)\end{array}$ & $\begin{array}{c}50.9 \\
\left(\mathrm{CHCl}_{3}\right)\end{array}$ & $\begin{array}{l}1.55(\mathrm{~d}, \mathrm{~J}=7.45,3 \mathrm{H}, \mathrm{Me}), 4.37(\mathrm{q}, \mathrm{J}=6.68 \\
\left.2 \mathrm{H}, \mathrm{NHCH}_{2} \mathrm{Ar}\right), 4.60(\mathrm{q}, \mathrm{J}=7.45,1 \mathrm{H}, 2-\mathrm{H}) \\
6.81(\mathrm{bs}, \mathrm{HH}, \mathrm{NH}) \\
466\left(\mathrm{M}^{+}, 10\right), 288(10), 206(85)\end{array}$ \\
\hline $8 d$ & 4 & 88 & $\begin{array}{c}142-143 \\
\left(\mathrm{CH}_{3} \mathrm{CN}\right)\end{array}$ & $\begin{array}{c}38.9 \\
\left(\mathrm{CHCl}_{3}\right)\end{array}$ & $\begin{array}{l}1.75(\mathrm{~d}, \mathrm{~J}=8.37,3 \mathrm{H}, \mathrm{Me}), 4.55(\mathrm{~d}, \mathrm{~J}=6.65 \\
2 \mathrm{H}, \text { pyridyl-CH } \mathrm{CH}_{2}, 4.80(\mathrm{q}, \mathrm{J}=8.37, \mathrm{lH}, 2-\mathrm{H}) \\
423\left(\mathrm{M}^{+}, 5\right), 162(33), 135(100)\end{array}$ \\
\hline $8 e$ & 9 & 62 & $\begin{array}{c}\text { 165-166 } \\
\text { (Hexane: } \\
\text { acetone=1:2) }\end{array}$ & $\begin{array}{c}32.0 \\
\left(\mathrm{CHCl}_{3}\right)\end{array}$ & $\begin{array}{l}1.65(\mathrm{~d}, \mathrm{~J}=7.14,3 \mathrm{H}, \mathrm{Me}), 4.40-4.60(\mathrm{~m}, 2 \mathrm{H}, \\
\left.\text { pyridyl } \mathrm{CH}_{2}\right), 4.75(\mathrm{q}, \mathrm{J}=7.14,1 \mathrm{H}, 2-\mathrm{H}), \\
6.87(\mathrm{bs}, \mathrm{HH}, \mathrm{NH}) \\
423\left(\mathrm{M}^{+}, 8\right), 315(25), 288(15), 261(8), \\
92(100)\end{array}$ \\
\hline $8 f$ & 3 & 65 & $\begin{array}{c}\text { 154-155 } \\
\text { (Hexane: } \\
\text { acetone }=1: 2 \text { ) }\end{array}$ & $\begin{array}{c}21.7 \\
\left(\mathrm{CHCl}_{3}\right)\end{array}$ & $\begin{array}{l}1.66(\mathrm{~d}, \mathrm{~J}=6.38,3 \mathrm{H}, \mathrm{Me}), 4.50(\mathrm{~d}, \mathrm{~J}=6.97, \\
\left.2 \mathrm{H}, \text { pyridyl } \mathrm{CH}_{2}\right), 4.80(\mathrm{q}, \mathrm{J}=6.38 \mathrm{lH}, 2-\mathrm{H}), \\
6.90(\mathrm{bs}, 1 \mathrm{H}, \mathrm{NH}) ; 423\left(\mathrm{M}^{+}, 8\right), 315(35), \\
289(18), 261(19), 120(35), 92(100)\end{array}$ \\
\hline
\end{tabular}


anhydrous potassium carbonate $(4.56 \mathrm{~g}, 33 \mathrm{mmol})$ was heated at reflux with stirring in dry acetonitrile $(50 \mathrm{~mL})$ until no $6 \mathrm{a}-\mathrm{f}$ could be detected by TLC. Potassium carbonate was removed by filtration and the solution was evaporated in vacuo. In case of $8 \mathrm{a}-\mathrm{c}$ the residue was crystallized and in case of $8 \mathrm{c}$ as well as $8 \mathrm{f}$ it was purified by column chromatoghraphy on silicagel (Table 2 ).

\section{Acknoledgement:}

The authors thank the National Science Foundation (OTKA-T034250) for financial support.

\section{References}

1 R. Buchanan, V. Sprancmanis and R.A. Partyka, J. Med. Chem. 12, 1001 (1969)

2 J. Lehman and F. Latanowicz, 'Arch. Pharm. 319, 278 (1986)

3 G. Bettoni, S. Ferorelli, F. Loiodice, N. Tangari, V. Tortorella, F. Gasparrini, D. Misiti and C. Villani, Chirality, 4, 193 (1992)

4 'R.E. Plowman, W.C. Stonebridge and J.N. Hawtree, Proc. Br. Crop. Prot. Conf.-Weeds, 1, 29 (1980), CA. 95, 56156j (1981)

5 T. Haga, K. Fujikawa, N. Sakashita and R. Nishiyama, J. Pesticide. Sci. 10(2), 257 (1985)

6 F. Bartha, V. Galamb, A. Repasi-Veres, T. Timar, J. Tompa, I. Fodor and K. Zsupan, Hung. Teljes HU. 47,525 (1989), CA. 112, 17758g (1990)

7 J. Tompa, F. Bartha, T. Timár, A. Répasi, V. Galamb, Ė. Füzi-Porkoláb, D. Miklós, Eur. Pat. 'Appl. EP 426,491 (1989), CA. 115, 114363 u (1991)

8 P.F. Bocion, P. Muehlethaler and P. Winternitz, Proc. Br. Crop. Prot. Conf.-Weeds, 1, 55 (1987), CA. 108, 126572e (1988)

9 A.R. Bell and A.S. Peddie, Proc. Br. Crop Prot. Conf.-Weeds, 1, 65 (1989), CA. 112, $212375 \mathrm{~g}$ (1990)

10 J. Amrein, A. Nyffeler and J. Rufener, Proc. Br. Crop. Prot. Conf.-Weeds, 1, 76 (1989), CA. 112, 173974e (1990)

11 -H.P. Huff, B. Buettner, E. Ebert and P. Langelueddeke, Proc. Br. Crop. Prot. Conf.-Weeds, 1, 717 (1989), CA. 112 173984h (1990)

12 J. Köver, T. Timar and J. Tompa, Synthesis, 11, 1124 (1994)

13 R. Handte, H. Schoenowsky, K. Bauer and H. Bieringer, Ger. Offen. DE 2,926,607. CA. 94, 156905a (1981)

14 CAChe Ver. 3.2 (Oxford Molecular Ltd., 1999)

15 K. Sato, N. Zakmazaki, Jap. Pat. 63 174, 952 CA 109, 230565m (1988) 